\title{
Association of CD31 and p53 With Survival of Ovarian Cancer Patients
}

\author{
LENE RASK ${ }^{1,2 \#}$, CLAUS K. HØGDALL ${ }^{3}$, SUSANNE K. KJAER ${ }^{2,3}$, LISE CHRISTENSEN ${ }^{1}$, \\ ALLAN JENSEN $^{2}$, JAN BLAAKAER ${ }^{4}$, IB JARLE CHRISTENSEN ${ }^{1}$ and ESTRID V.S. HØGDALL ${ }^{1}$ \\ ${ }^{1}$ Department of Pathology, Herlev-og Gentofte Hospital, University of Copenhagen, Herlev, Denmark; \\ ${ }^{2}$ Department of Virus, Lifestyle and Genes, Danish Cancer Society Research Center, Copenhagen, Denmark; \\ ${ }^{3}$ Department of Gynaecology, Rigshospitalet, University of Copenhagen, Copenhagen, Denmark; \\ ${ }^{4}$ Department of Gynecology and Obstetrics, Odense University Hospital, Odense, Denmark
}

\begin{abstract}
Background/Aim: New markers for ovarian cancer are needed. This study aimed to examine the expression of tumour cell p53 and endothelial cell CD31 proteins and correlate them to clinicopathological factors. Patients and Methods: Expression of proteins was immunohistochemically assessed using tissue sections from 585-599 ovarian cancer patients from the Danish MALOVA study. Results: High CD31 expression was found in poorly differentiated tumours $(p=0.0006)$, and high $p 53$ expression was found in poorly differentiated cancers $(p<0.0001)$, high clinical stage $(p<0.0001)$, non-radical surgery $(p<0.0001)$ and high serum $C A-125$ values $(p<0.0001)$. CD31 expression showed no prognostic survival value, but high hazard ratios were found for patients with high p53 expression $(H R=2.313, p<0.0001)$. An interaction was found between p53 and stage of cancer, suggesting a prognostic impact of p53 in low-stage, but not in advancedstage cancer. Conclusion: More than 5\% of p53 tissue expression may predict shorter survival of ovarian cancer patients and may be useful for predicting the risk of disease progression in low-stage patients following primary surgery. CD31 has no strong prognostic value.
\end{abstract}

Cancer of the ovaries is the ninth most frequently diagnosed cancer in women (1). Due to the lack of symptoms, most patients are diagnosed at a late clinical stage, efficaciously reducing survival. Six-year survival rate of Danish ovarian

\#Current affiliation: Department of Clinical Neurophysiology, Rigshospitalet - Glostrup, Glostrup, Denmark.

Correspondence to: Estrid Høgdall, Department of Pathology, Herlev- og Gentofte Hospital, Herlev Ringvej 75, 2730 Herlev, Denmark. Tel: +45 38689132, e-mail: estrid.hoegdall@regionh.dk

Key Words: p53, CD31, ovarian cancer. and peritoneal cancer patients (years 2005-2011) ranged from $80 \%$ for stage I patients to $10.8 \%$ for stage IV patients (www.dgcg.dk). Therefore, biomarkers of molecular changes specific for ovarian cancer (OC) development and progression are required in order to stratify patients to risk groups.

OCs vary in clinical behaviour and are classified into histological subtypes (serous, mucinous, endometrioid and clear cell) (2). Negative prognostic indicators for OC include advanced FIGO stage, high tumour grade, incomplete surgical tumour resection and mutations in proto-oncogenes Kirsten rat sarcoma virus $(K R A S), \mathrm{V}-\mathrm{RAF}$ murine sarcoma viral oncogene homolog $\mathrm{B} 1(B R A F)$ and tumour suppressor p53 (TP53) (3-6). Other suggested prognostic indicators are markers for angiogenesis (7).

Synthesis and rearrangement of blood vessels are necessary during massive tumour growth, where the cells depend on oxygen and nutrients as well as discharge of waste substances by the blood stream, and the majority of tissue cells are found within $100 \mu \mathrm{m}$ from blood supply (8). Angiogenesis in tumour growth can be studied using immunohistochemistry, which can detect endothelial cellspecific proteins, such as platelet endothelial cell adhesion molecule-1 (PECAM-1/CD31) (9).

CD31 protein is present on endothelial cells in intratumoural microvessels of cancers such as OC (10). In OC, conflicting results have been obtained regarding the correlation of high CD31 expression with advanced disease and poor survival (11-18).

An important gene involved in cell-cycle regulation, DNA repair and apoptosis is the TP53 gene located on chromosome 17 and encoding the tumour suppressor protein p53 $(19,20)$. TP53 is a frequently mutated gene in cancer including OC $(6,20)$. Most mutations in TP53 lead to a nonfunctional p53 protein with increased stability that may be detected by immunohistochemistry as an accumulation of p53 protein in tumour cells (20). 
The objective of this study was to examine vessel CD31 expression and the tumour cell expression of $\mathrm{p} 53$ protein in a large number of well-defined histologically different OC tissue sections from a cohort of Danish patients with OC and correlate these to clinicopathological factors.

\section{Patients and Methods}

Patient characteristics. Patient data set were retrieved from the MALOVA (MALignant OVArian) cancer study $(21,22)$. MALOVA is a Danish multicenter study, designed to identify risk factors and prognostic markers for OC. A total of 633 tissue samples obtained from the primary operation of women diagnosed with $\mathrm{OC}$ were used (23-25). The sample material was collected from the gynaecological departments of 18 Danish hospitals. OC cases were histologically graded as well, moderately or poorly differentiated based on hematoxylin and eosin (HE)-stained tissue sections. Clinically, they represented FIGO stages I-IV according to the guidelines from the International Federation of Gynaecologists and Obstetricians. The OCs were histologically subclassified as carcinoma NOS (not otherwise specified), serous well/moderately differentiated, serous poorly differentiated, mucinous, endometrioid or clear-cell carcinoma on one side and non-epithelial ovarian tumours on the other (Table I). The study was performed following permission by the Scientific Ethical Committees (study reference number KF01-384/95).

Immunohistochemical staining of CD31 and p53. Tissues were fixed in formalin at room temperature, embedded in paraffin and used for tissue array analyses with $2 \mathrm{~mm}$ (diameter) large tissue cores obtained from each paraffin block. Immunohistochemical staining of $2 \mu \mathrm{m}$ tissue sections was performed according to the manufacturer's recommendations (Dako A/S, Glostrup, Denmark). After deparaffinization and antigen-retrieval, the tissue sections were incubated with the monoclonal CD31 (clone JC70A, Dako) antibody (diluted 1:40) or the monoclonal antibody DO7 (Dako) against p53 (diluted 1:400) for $1 \mathrm{~h}$ at room temperature according to the manufacturer's recommendations (23). The DO7 antibody has been reported to detect both wild type and mutated p53 protein (26). Due to lack of material, the absence of sufficient tumour tissue or inadequate tissue, 34-48 samples were excluded.

Scoring of immunohistochemistry. Samples from 599 and 585 OCs were scored for CD31 and p53 expression, respectively. Slides were scored semi-quantitatively by two experienced observers. Only distinct CD31 membrane staining of endothelial cells and nuclear p53 staining of ovarian tumour cells were considered. CD31 immunoreactivity was categorized as negative (0), weak (1), moderate (2) or strong (3) and p53 staining was calculated as the percentage of positive tumour cell nuclei and transformed into a two-tiered scale - negative for no p53 expression and positive for p53 expression above 5\% (23). p53 was scored dichotomized with $5 \%$ as the threshold.

Statistical analysis. The Chi-square test was used to calculate any differences in CD31 and p53 expression between tumour types, FIGO stages or differentiation grades. Any association between continuous variables (CA-125) and other countable variables was evaluated using the Kruskal-Wallis test. Univariate survival analysis was performed with Kaplan-Meier and Log rank significance test to evaluate any prognostic value of CD31 and p53 expression.
Table I. Clinical characteristics of ovarian cancer patients.

\begin{tabular}{|c|c|c|}
\hline \multirow[t]{2}{*}{ Characteristics } & \multicolumn{2}{|c|}{ Ovarian cancer } \\
\hline & $\mathrm{N}$ & $(\%)$ \\
\hline Number & 633 & \\
\hline FIGO stage & 633 & \\
\hline I & 180 & $(28)$ \\
\hline II & 66 & (10) \\
\hline III & 332 & $(53)$ \\
\hline IV & 55 & (9) \\
\hline Histological tumour type & 633 & \\
\hline Carcinoma NOS & 43 & (7) \\
\hline Serous well /moderate diff adenocarcinoma & 241 & $(38)$ \\
\hline Serous poorly diff adenocarcinoma & 137 & $(22)$ \\
\hline Mucinous adenocarcinoma & 54 & (9) \\
\hline Endometrioid adenocarcinoma & 84 & (13) \\
\hline Non-epithelial ovarian tumours & 27 & (4) \\
\hline Clear-cell carcinoma & 47 & (7) \\
\hline Chemotherapy (681) & 633 & \\
\hline No chemotherapy & 162 & $(26)$ \\
\hline Non-platinum chemotherapy & 55 & (9) \\
\hline Platinum-based chemotherapy & 416 & (66) \\
\hline Differentiation grade & 632 & \\
\hline Well & 155 & $(25)$ \\
\hline Moderate & 229 & (36) \\
\hline Poorly & 248 & (39) \\
\hline Radicality of surgery & 633 & \\
\hline Radical surgery & 247 & (39) \\
\hline Non-radical surgery & 386 & (61) \\
\hline \multirow[t]{2}{*}{ Serum CA-125 } & 422 & \\
\hline & Median & $\begin{array}{l}\text { (minimum, } \\
\text { maximum) }\end{array}$ \\
\hline Serum CA-125 value & 378 & $(2,165150)$ \\
\hline
\end{tabular}

NOS: Not otherwise specified; Diff: differentiation.

Univariate Cox proportional hazard ratios (HRs) were calculated to analyse impact of CD31 and p53 expression/level on survival. Multivariate analysis was carried out using Cox proportional HRs stratified by chemotherapy group and adjusted for markers, FIGO stage, differentiation grade, histological tumour type, age and radicality of surgery and tests for interaction were also performed. HRs are provided with $95 \%$ confidence intervals (CI). Overall survival (OS) and disease specific survival (DSS) were defined as time from primary surgery until death of any cause or death from OC, respectively. Progression-free survival (PFS) was calculated from the date of surgery to the date of documented disease progression (clinical and/or biochemical). Statistical significance was considered at the $5 \%$ probability level in all cases $(p \leq 0.05)$. All statistical calculations were performed with SAS (v9.4, SAS Institute, Cary, NC, USA).

\section{Results}

Clinical characteristics. A total of 633 patients diagnosed with OC were included in this study (Table I). As anticipated, most of the OC cases had well/moderately 
Table II. Univariate analysis of the relationship between CD31 expression, p53 protein expression and clinical characteristics ${ }^{d}$.

\begin{tabular}{|c|c|c|c|c|c|c|c|c|}
\hline \multirow[t]{3}{*}{ Clinical variable } & \multicolumn{4}{|c|}{ Endothelial CD31 presence ${ }^{a}$} & \multicolumn{4}{|c|}{ p53 presence $^{b}$} \\
\hline & 0 & 1 & 2 & 3 & $p$-Value & $\leq 5 \%$ & $>5 \%$ & $p$-Value \\
\hline & \multicolumn{4}{|c|}{$\mathrm{N}(\%)$} & \multicolumn{4}{|c|}{$\mathrm{N}(\%)$} \\
\hline FIGO stagec & 123 & 329 & 130 & 17 & 0.2569 & 327 & 258 & $<0.0001$ \\
\hline I & $40(23)$ & $83(48)$ & $45(26)$ & $5(3)$ & & $126(78)$ & $35(22)$ & \\
\hline II & $13(21)$ & $35(57)$ & $12(19)$ & $2(3)$ & & $43(68)$ & $20(32)$ & \\
\hline III & $61(20)$ & $173(56)$ & $67(22)$ & $10(3)$ & & $134(44)$ & $174(57)$ & \\
\hline IV & $9(17)$ & $38(72)$ & $6(11)$ & $0(0)$ & & $24(45)$ & $29(55)$ & \\
\hline Histological tumour type $\mathrm{e}^{\mathrm{c}}$ & 123 & 329 & 130 & 17 & $<0.0001$ & $327^{\mathrm{d}}$ & $258^{\mathrm{d}}$ & $<0.0001$ \\
\hline Carcinoma NOS & $3(8)$ & $15(39)$ & $19(49)$ & $2(5)$ & & $21(53)$ & $19(48)$ & \\
\hline Serous adenocarcinoma Well/moderate Diff grade & $62(27)$ & $141(62)$ & $22(10)$ & $1(0)$ & & $122(53)$ & $107(47)$ & \\
\hline Serous adenocarcinoma Poorly diff grade & $26(20)$ & $77(60)$ & $23(18)$ & $2(2)$ & & $40(30)$ & $95(70)$ & \\
\hline Mucinous adenocarcinoma & $12(23)$ & $23(44)$ & $13(25)$ & $4(8)$ & & $44(86)$ & $7(14)$ & \\
\hline Endometrioid adenocarcinoma & $5(6)$ & $32(39)$ & $39(48)$ & $6(7)$ & & $59(71)$ & $24(29)$ & \\
\hline Non-epithelial ovarian tumours & $8(31)$ & $11(42)$ & $6(23)$ & $1(4)$ & & - & - & \\
\hline Clear-cell carcinoma & $7(15)$ & $30(65)$ & $8(17)$ & $1(2)$ & & $41(87)$ & $6(13)$ & \\
\hline Differentiation grade ${ }^{c}$ & 123 & 329 & 130 & 17 & 0.0006 & 326 & 258 & $<0.0001$ \\
\hline Well & $44(31)$ & $76(53)$ & $24(17)$ & $0(0)$ & & $104(74)$ & $37(26)$ & \\
\hline Moderate & $38(17)$ & $135(61)$ & $41(19)$ & $8(4)$ & & $129(60)$ & $87(40)$ & \\
\hline Poorly & $41(18)$ & $118(51)$ & $65(28)$ & $9(4)$ & & $93(41)$ & $134(59)$ & \\
\hline Radicality of surgery ${ }^{\mathrm{c}}$ & 123 & 329 & 130 & 17 & 0.9123 & 327 & 258 & $<0.0001$ \\
\hline Radical surgery & $50(21)$ & $127(54)$ & $54(23)$ & $6(3)$ & & $167(74)$ & $59(26)$ & \\
\hline Non-radical surgery & $73(20)$ & $202(56)$ & $76(21)$ & $11(3)$ & & $160(45)$ & $199(55)$ & \\
\hline p53 expression ${ }^{b, c}$ & 110 & 316 & 124 & 16 & 0.4742 & & & \\
\hline$\leq 5 \%$ & $61(19)$ & $174(55)$ & $68(22)$ & $12(4)$ & & & & \\
\hline$>5 \%$ & $49(20)$ & $142(57)$ & $56(22)$ & $4(2)$ & & & & \\
\hline \multirow[t]{2}{*}{ Serum CA- $125^{\mathrm{c}}$} & 90 & 222 & 81 & 12 & 0.6556 & 227 & 167 & $<0.0001$ \\
\hline & \multicolumn{4}{|c|}{ Median (min, max) } & \multicolumn{4}{|c|}{ Median (min, $\max )$} \\
\hline Serum CA-125 value & 287 & 471 & 332 & 134 & & 261 & 548 & \\
\hline & $(2,165150)$ & $(4,13529)$ & $(6,32659)$ & $(14,3068)$ & & $(2,165150)$ & $(4,32659)$ & \\
\hline
\end{tabular}

min: Minimum; Max: maximum; NOS: not otherwise specified; Diff: Differentiation. a 0 , negative; 1 , weak; 2 , moderate; 3 , high. ${ }^{b}$ Percentage positive

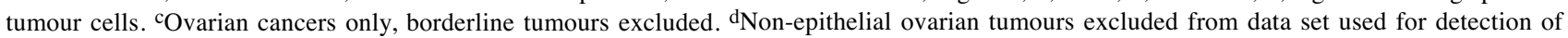
p53 protein.

differentiated or poorly differentiated serous adenocarcinoma $(60 \%)$. With respect to FIGO stage, OCs were mainly diagnosed at the later clinical stage III (53\%). According to the Danish gynaecology cancer group (DGCG) guidelines, most OC patients, except for a subgroup of low stage patients, are treated with platinum-based anti-cancer drugs (66\% in our data set), often carboplatin in combination with a taxane (www.dgcg.dk).

CD31 expression and clinical characteristics. Detection of CD31 immunoreactivity and its association with clinicopathological features are shown in Table II. No significant association was found between CD31 staining of OCs on one side and FIGO stage $(p=0.2569)$, radicality of surgery $(p=0.9123)$ or serum level of CA-125 $(p=0.6556)$ on the other. On the contrary, a significant inverse correlation was found between differentiation grade of tumour and
CD31 expression ( $p=0.0006)$ corresponding to an increase in CD31 expression in poorly differentiated tumours (2-3, $32 \%)$ as opposed to well differentiated carcinomas (2-3, $17 \%$ ). The CD31 expression levels in OCs were not the same across different histological tumour types $(p<0.0001)$. Higher expression of CD31 was found in carcinoma NOS and endometrioid carcinomas (2-3, 54-55\%), and lower levels were seen in serous well/moderately differentiated and poorly differentiated, mucinous, clear-cell and non-epithelial tumours $(0-1,67-89 \%)$ (Table II).

p53 and clinical characteristics. Significant differences in high and low detection scores for p53 in OC were seen in relation to FIGO stage $(p<0.0001)$ and differentiation grade of the tumours $(p<0.0001)$ (Table II). High scores of p53 protein were found in advanced FIGO stages III-IV (56\%) compared to low FIGO stages I-II (25\%) and in poorly 
Table III. Univariate analysis of overall survival (OS), disease-specific survival (DSS) and progression-free survival (PFS) with respect to CD31 expression (1,2,3), expression of p53 protein (>5\%) compared to absence of CD31 protein $(0)$ or p53 protein $(\leq 5 \%)^{a}$.

\begin{tabular}{|c|c|c|c|c|c|c|c|c|c|}
\hline \multirow[t]{2}{*}{ Covariate } & HR & $95 \% \mathrm{CI}$ & $p$-Value & $\mathrm{HR}$ & $95 \% \mathrm{CI}$ & $p$-Value & HR & $95 \% \mathrm{CI}$ & $p$-Value \\
\hline & \multicolumn{3}{|c|}{ OS } & \multicolumn{3}{|c|}{ DSS } & \multicolumn{3}{|c|}{ PFS } \\
\hline \multicolumn{10}{|l|}{$\mathrm{CD}_{1} 1$ expression $^{\mathrm{b}}(\mathrm{N}=599)$} \\
\hline 1 vs. 0 & 1.071 & $0.844-1.358$ & 0.5724 & 1.142 & $0.872-1.494$ & 0.3346 & 1.189 & $0.905-1.561$ & 0.2142 \\
\hline 2 vs. 0 & 0.948 & $0.712-1.263$ & 0.7174 & 1.004 & $0.727-1.386$ & 0.9817 & 1.018 & $0.734-1.414$ & 0.9130 \\
\hline 3 vs. 0 & 0.879 & $0.482-1.603$ & 0.6733 & 0.959 & $0.495-1.859$ & 0.9009 & 0.989 & $0.494-1.981$ & 0.9752 \\
\hline \multicolumn{10}{|l|}{ p53 protein expression ${ }^{\mathrm{c}}(\mathrm{N}=585)$} \\
\hline$>5 \%$ vs. $\leq 5 \%$ & 2.011 & $1.660-2.437$ & $<0.0001$ & 2.313 & $1.868-2.866$ & $<0.0001$ & 2.446 & $1.970-3.038$ & $<0.0001$ \\
\hline \multicolumn{10}{|l|}{ FIGO stage $(\mathrm{N}=633)$} \\
\hline II $v s$. I & 2.215 & $1.518-3.231$ & $<0.0001$ & 3.514 & $2.126-5.808$ & $<0.0001$ & 4.082 & $2.651-6.285$ & $<0.0001$ \\
\hline III $v s$. I & 5.695 & $4.387-7.393$ & $<0.0001$ & 9.875 & $6.738-14.474$ & $<0.0001$ & 9.063 & $6.457-12.720$ & $<0.0001$ \\
\hline IV vs. I & 13.777 & $9.553-19.870$ & $0<0.0001$ & 21.250 & $13.371-33.770$ & $<0.0001$ & 16.492 & $10.292-26.429$ & $<0.0001$ \\
\hline \multicolumn{10}{|l|}{ Histological tumour type $(\mathrm{N}=633)$} \\
\hline $\begin{array}{l}\text { Clear cell } v s \text {. Serous } \\
\text { adenocarcinoma poorly diff grade } \\
\text { Endometrioid } v s \text {. Serous }\end{array}$ & 0.346 & $0.225-0.532$ & $<0.0001$ & 0.369 & $0.229-0.594$ & $<0.0001$ & 0.301 & $0.189-0.481$ & $<0.0001$ \\
\hline adenocarcinoma poorly diff grade & 0.373 & $0.269-0.518$ & $<0.0001$ & 0.333 & $0.227-0.487$ & $<0.0001$ & 0.314 & $0.220-0.448$ & $<0.0001$ \\
\hline $\begin{array}{l}\text { Mucinous vs Serous adenocarcinoma } \\
\text { poorly diff grade }\end{array}$ & 0.316 & $0.208-0.480$ & $<0.0001$ & 0.291 & $0.176-0.479$ & $<0.0001$ & 0.230 & $0.140-0.379$ & $<0.0001$ \\
\hline \multicolumn{10}{|l|}{ NOS vs. Serous adenocarcinoma } \\
\hline \multicolumn{10}{|l|}{ Non-epithelial ovarian tumours $v s$. } \\
\hline Serous adenocarcinoma poorly diff grade & le 0.550 & $0.335-0.902$ & 0.0179 & 0.600 & $0.350-1.029$ & 0.0636 & 0.413 & $0.237-0.720$ & 0.0018 \\
\hline & \multicolumn{3}{|c|}{ Serous adenocarcinoma well/moderate } & 0.655 & $0.514-0.835$ & 0.0006 & 0.598 & $0.472-0.757$ & $<0.0001$ \\
\hline \multicolumn{10}{|l|}{ Differentiation grade $(\mathrm{N}=632)$} \\
\hline Well vs. Poorly & 0.462 & $0.359-0.594$ & $<0.0001$ & 0.378 & $0.281-0.508$ & $<0.0001$ & 0.400 & $0.300-0.535$ & $<0.0001$ \\
\hline Moderate vs. Poorly & 0.689 & $0.560-0.847$ & 0.0004 & 0.657 & $0.525-0.824$ & 0.0003 & 0.645 & $0.513-0.812$ & 0.0002 \\
\hline \multicolumn{10}{|l|}{ Radicality of surgey $(\mathrm{N}=633)$} \\
\hline Non-radical surgery $v s$. radical surgery & 4.531 & $3.650-5.624$ & $<0.0001$ & 6.949 & $5.248-9.203$ & $<0.0001$ & 7.041 & $5.396-9.188$ & $<0.0001$ \\
\hline \multicolumn{10}{|l|}{ Serum CA-125 (N=422) } \\
\hline 2-fold difference & 1.170 & $1.125-1.217$ & $<0.0001$ & 1.192 & $1.141-1.246$ & $<0.0001$ & 1.195 & $1.144-1.248$ & $<0.0001$ \\
\hline
\end{tabular}

NOS: Not otherwise specified; Diff: differentiation. ${ }^{a}$ Ovarian cancers only, borderline tumours excluded. b0, negative; 1 , weak; 2 , moderate; 3 ,

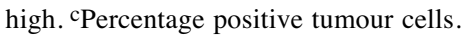

differentiated tumours (59\%) compared to well differentiated tumours $(26 \%)$. High and low expression of p53 in OC were not evenly distributed among different histological tumour subtypes $(p<0.0001)$. High levels were found in serous poorly differentiated carcinomas $(70 \%,>5 \%)$ and low levels in mucinous $(86 \%, \leq 5 \%)$ and clear-cell carcinomas $(87 \%$, $\leq 5 \%$ ). Moreover, significantly higher p53 tumour levels were detected in patients with residual tumour after primary surgery $(55 \%)$ as opposed to radically operated patients (26\%) and in patients with high CA-125 serum levels (median $548 \mathrm{U} / \mathrm{ml}$ ) as opposed to patients with low CA-125 levels (median $261 \mathrm{U} / \mathrm{ml})(p<0.0001)$.

Clinicopathological variables and survival. At follow-up, 479 patients with OC had died (75.7\%), 426 from OC. Median follow-up time was 177.9 months (range=153.3-205.7 months).
Univariate proportional hazard regression analysis of the patient data (599 patients) with respect to OS, DSS and PFS showed no prognostic value of CD31 expression $(p>0.05)$ (Table III). Significantly higher HRs were found for OC patients with high detected levels of the p53 protein (HR $\mathrm{OS}=2.011, \quad p<0.0001 ; \quad \mathrm{DSS}=2.313, \quad p<0.0001$ and $\mathrm{PFS}=2.446, p<0.0001)$. Significantly higher HRs were found for patients with advanced FIGO stage, compared to low FIGO stage (HR OS FIGO IV vs. I 13.777, $p<0.0001$; HR DSS FIGO IV $v s$. I 21.250, $p<0.0001$; HR PFS FIGO IV vs. I 16.492, $p<0.0001)$. Compared to serous poorly differentiated adenocarcinomas, clear cell $(p<0.0001)$, endometrioid $(p<0.0001)$ and mucinous adenocarcinomas $(p<0.0001)$ as well as serous well/moderately differentiated adenocarcinomas ( $p \leq 0.0001-0.0006)$ showed significantly lower HRs with respect to overall, disease specific survival 
Table IV. Multivariate analysis of overall survival (OS), disease-specific survival (DSS) and progression-free survival (PFS) of ovarian cancer patients $(N=379)^{\mathrm{a}, \mathrm{b}, \mathrm{f}}$

\begin{tabular}{|c|c|c|c|c|c|c|c|c|c|}
\hline \multirow[t]{2}{*}{ Clinical variable } & HR & $95 \% \mathrm{CI}$ & $p$-Value & HR & $95 \% \mathrm{CI}$ & $p$-Value & HR & $95 \% \mathrm{CI}$ & $p$-Value \\
\hline & \multicolumn{3}{|c|}{ OS } & \multicolumn{3}{|c|}{ DSS } & \multicolumn{3}{|c|}{ PFS } \\
\hline Endothelial CD31 expression ${ }^{\mathrm{c}}$ & & & 0.9809 & & & 0.9596 & & & 0.9521 \\
\hline 1 vs. 0 & 0.944 & $0.682-1.306$ & & 0.945 & $0.671-1.332$ & & 0.922 & $0.666-1.277$ & \\
\hline 2 vs. 0 & 0.998 & $0.663-1.502$ & & 0.974 & $0.637-1.489$ & & 0.983 & $0.652-1.484$ & \\
\hline 3 vs. 0 & 0.960 & $0.399-2.308$ & & 0.797 & $0.329-1.935$ & & 0.873 & $0.366-2.086$ & \\
\hline p53 protein presenced & & & 0.0339 & & & 0.0002 & & & $<0.0001$ \\
\hline$>5 \%$ vs. $\leq 5 \%$ & 1.341 & $1.022-1.758$ & & & & & & & \\
\hline FIGO stage & & & $<0.0001$ & & & $<0.0001$ & & & $<0.0001$ \\
\hline II $v s . \mathrm{I}$ & 1.846 & $0.838-4.066$ & & & & & & & \\
\hline III vs. I & 4.411 & $2.128-9.144$ & & & & & & & \\
\hline IV vs. I & 7.508 & $3.389-16.635$ & & & & & & & \\
\hline \multicolumn{10}{|l|}{ Interaction } \\
\hline p53 protein expression $x$ FIGO stage ${ }^{e}$ & & & & & & 0.0037 & & & 0.0043 \\
\hline$\leq 5 \%$ vs. $>5 \%$ at FIGO stage I & & & & 0.110 & $0.035-0.348$ & & 0.146 & $0.058-0.369$ & \\
\hline$\leq 5 \%$ vs. $>5 \%$ at FIGO stage II & & & & 0.330 & $0.117-0.932$ & & 0.649 & $0.264-1.597$ & \\
\hline$\leq 5 \%$ vs. $>5 \%$ at FIGO stage III & & & & 0.851 & $0.608-1.191$ & & 0.872 & $0.636-1.197$ & \\
\hline$\leq 5 \%$ vs. $>5 \%$ at FIGO stage IV & & & & 0.599 & $0.302-1.192$ & & 0.842 & $0.426-1.664$ & \\
\hline Histological tumour type & & & 0.2369 & & & 0.0648 & & & 0.1013 \\
\hline $\begin{array}{l}\text { Clear cell } v s . \text { Serous } \\
\text { adenocarcinoma poorly diff grade } \\
\text { Endometrioid } v s . \text { Serous }\end{array}$ & 1.563 & $0.811-3.014$ & & 2.345 & $1.171-4.694$ & & 1.765 & $0.917-3.396$ & \\
\hline adenocarcinoma poorly diff grade & 0.980 & $0.607-1.582$ & & 1.123 & $0.667-1.893$ & & 0.916 & $0.564-1.488$ & \\
\hline $\begin{array}{l}\text { Mucinous } v s . \text { Serous adenocarcinoma } \\
\text { poorly diff grade }\end{array}$ & 1.095 & $0.500-2.395$ & & 1.085 & $0.503-2.343$ & & 1.120 & $0.514-2.441$ & \\
\hline NOS vs. Serous adenocarcinoma & & & & & & & & & \\
\hline poorly diff grade & 1.131 & $0.653-1.959$ & & 1.285 & $0.716-2.306$ & & 1.002 & $0.574-1.747$ & \\
\hline $\begin{array}{l}\text { Serous adenocarcinoma well/moderate } \\
\text { diff grade } v s \text {. Serous adenocarcinoma }\end{array}$ & & & & & & & & & \\
\hline poorly diff grade & 0.783 & $0.577-1.064$ & & 0.836 & $0.606-1.153$ & & 0.752 & $0.552-1.025$ & \\
\hline Radicality of surgery & & & 0.0001 & & & 0.0001 & & & $<0.0001$ \\
\hline Non-radical surgery $v s$. radical surgery & 2.588 & $0.585-4.225$ & & 2.795 & $1.651-4.732$ & & 2.811 & $1.714-4.610$ & \\
\hline Age & & & 0.2211 & & & 0.0126 & & & 0.2134 \\
\hline 10-year interval & 1.087 & $0.951-1.242$ & & 1.202 & $1.040-1.390$ & & 1.089 & $0.952-1.245$ & \\
\hline Serum CA-125 & & & 0.8926 & & & 0.9969 & & & 0.6453 \\
\hline 2-fold difference & 0.996 & $0.940-1.056$ & & 1.000 & $0.941-1.063$ & & 0.986 & $0.931-1-045$ & \\
\hline
\end{tabular}

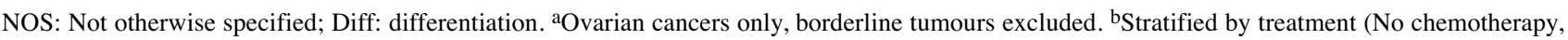
non-platinum chemotherapy or platinum-based chemotherapy). Adjusted for all variables (markers, FIGO stage, differentiation grade, histological

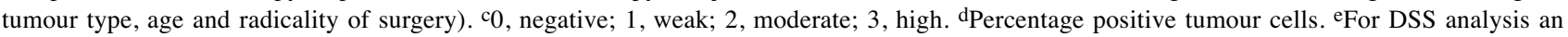
effect modulation was seen for p53 protein expression and FIGO stage. ${ }^{\mathrm{f}}$ Differentiation grade not included as serous adenocarcinoma is separated into high grade and low-grade carcinomas.

and PFS. A significantly lower HR for non-epithelial ovarian tumours compared to serous poorly differentiated adenocarcinomas was also found with respect to OS and PFS ( $p=0.0179$ and $p=0.0018)$.

Low HRs were found in well differentiated OC compared to poorly differentiated tumours (HR OS $=0.462, p<0.0001$; HR DSS $=0.378, p<0.0001$; HR PFS $=0.400, p<0.0001)$ and high HRs were found for patients with residual tumour after primary surgery $(p<0.0001)$ and patients with high levels of serum CA-125 $(p<0.0001)$ (Table III).

In accordance with the hazard regression results, the Kaplan-Meier estimates of DSS and PFS, revealed a significantly shorter survival of OC patients with high levels of p53 protein compared to patients with low levels of p53 $(p<0.0001)$ (Figure 1B and D). Categorising patients according to CD31 expression levels $(0,1,2,3)$ did not show 
A

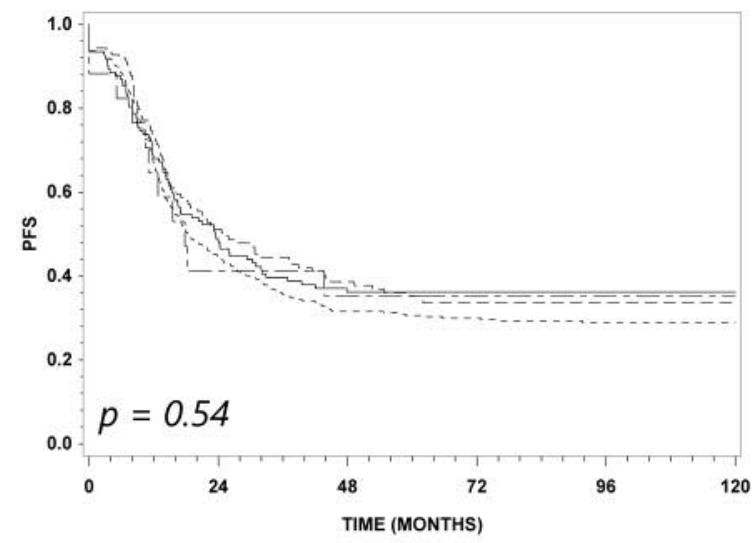

B

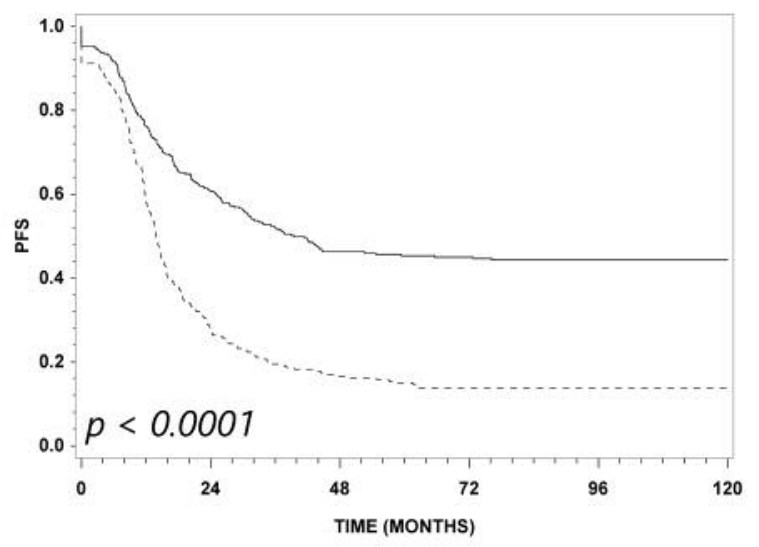

$\begin{array}{llllll}\text { EVENTS } & \text { PATIENTS AT RISK } & & & \\ 161 & 320 & 192 & 143 & 134 & <5 \%\end{array}$ 191

41

33

$>5 \%$

\section{C}

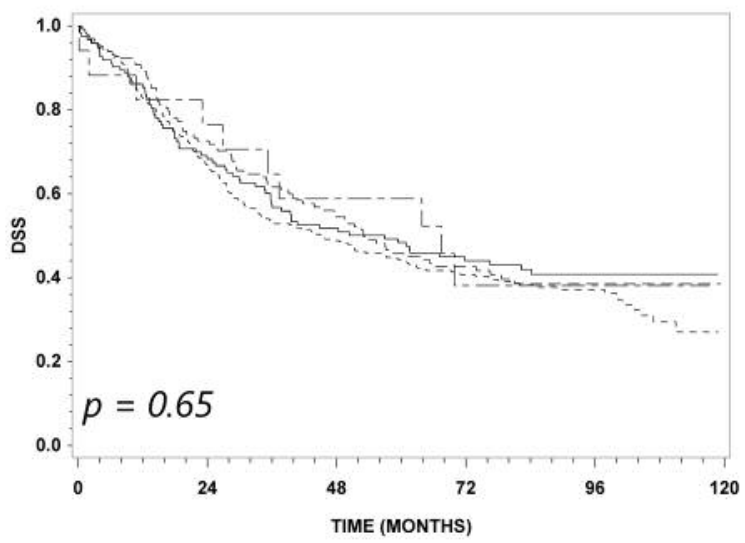

D

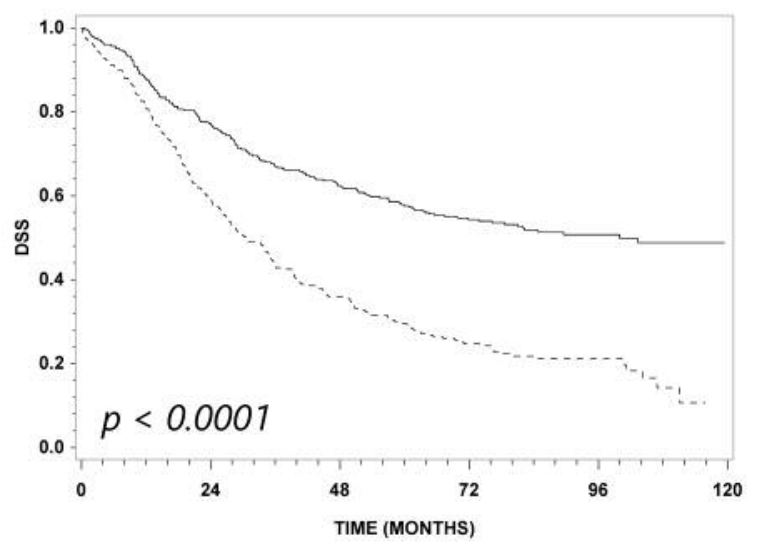

$\begin{array}{lrlll}\text { PATIENTS AT RISK } & & & & \\ 327 & 251 & 198 & 154 & <5 \%\end{array}$

$258 \quad 151$

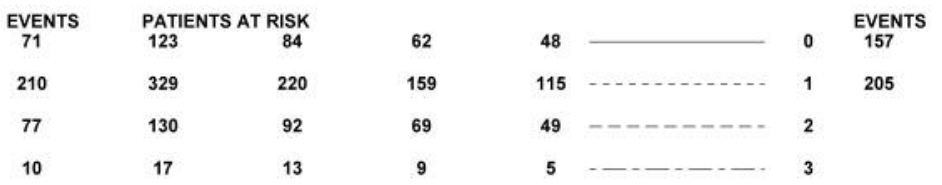

Figure 1. Kaplan-Meier plot of progression-free survival (PFS) (A-B) and disease-specific survival (DSS) (C-D) based on CD31 expression (A, C) and $p 53$ levels $(B, D)$. A significant improved survival was found for patients with low levels of $p 53(B, D)$.

any significant differences in DSS or PFS $(p=0.54$ and $p=0.65$ ) (Figure 1A and C).

Multivariate survival analysis. Independent prognostic indicators are listed in Table IV. As expected, FIGO stage (HR OS IV vs. I 7.508, $p<0.0001$ ) and radicality of surgery (HR OS $=2.588, p=0.0001$; HR DSS $=2.795, p=0.0001$; HR $\mathrm{PFS}=2.811, p<0.0001)$ remained prognostic predictors in the multivariate analysis. Also, increasing age at diagnosis correlated with decreased DSS (HR=1.202, $p=0.0126)$, but not with OS and PFS.

In the OS, DSS and PFS analysis, p53 was identified as an independent prognostic factor $(p=0.0339, p=0.0002$ and $p<0.0001$, respectively), and a significant interaction was found between p53 protein expression and FIGO stage ( $p=0.0037$, DSS and $p=0.0043$, PFS), suggesting a high 
A

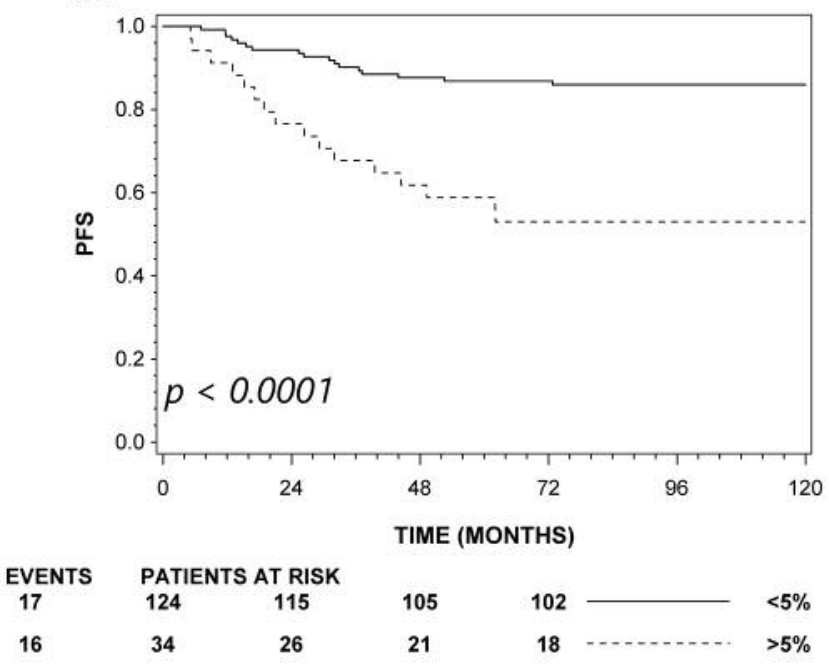

B

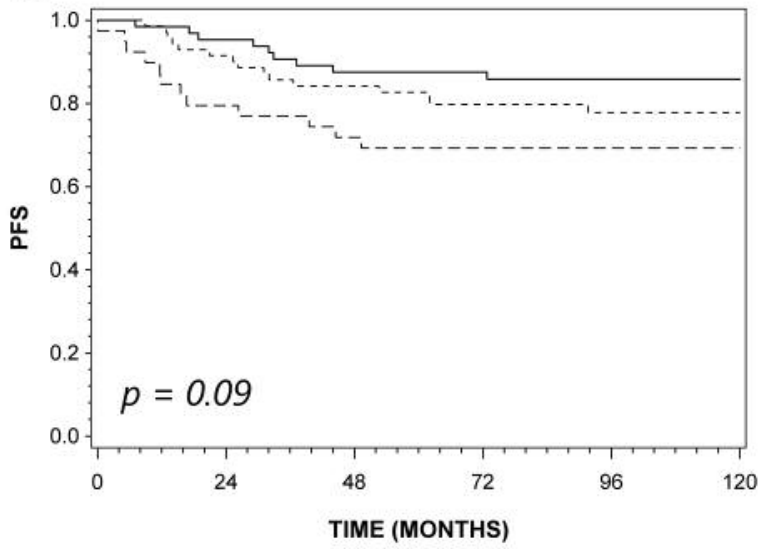

\begin{tabular}{|c|c|c|c|c|c|c|}
\hline$\underset{9}{\text { EVENTS }}$ & $\begin{array}{r}\text { PAT } \\
65\end{array}$ & $\begin{array}{c}\text { T RISK } \\
61\end{array}$ & 56 & 52 & - & HIGH \\
\hline 16 & 71 & 64 & 57 & 54 & -. & MOD \\
\hline 11 & 39 & 31 & 28 & 26 & -------- & LOW \\
\hline
\end{tabular}

Figure 2. Kaplan-Meier plot of progression-free survival (PFS) of stage I patients based on p53 level (A) and grade (B). A significantly improved survival was found for stage I patients with low level of p53 compared to stage I patients with high levels of p53.

Table V. Relation between overexpression of p53 protein and tumour progression for FIGO stage I ovarian cancer patients without adjuvant chemotherapya.

\begin{tabular}{|c|c|c|c|}
\hline & \multicolumn{3}{|c|}{ p53 presence ${ }^{b}$} \\
\hline & $\leq 5 \%$ & $>5 \%$ & $p$-Value \\
\hline & \multicolumn{3}{|c|}{$\mathrm{N}(\%)$} \\
\hline Progression $^{\mathrm{c}}$ & 91 & 18 & 0.0005 \\
\hline No progression & $81(89)$ & $10(11)$ & \\
\hline Progression & $10(56)$ & $8(44)$ & \\
\hline
\end{tabular}

aOvarian cancers only, borderline tumours excluded. bPercentage

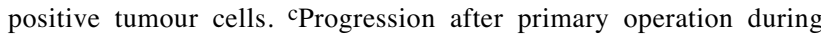
follow-up verified by an elevated CA-125 level, ultrasound scan and/or pelvic examination.

impact of $\mathrm{p} 53$ on predicting prognosis in low stage patients (p53 $\leq 5 \%$ vs. $>5 \%$ ) (HR DSS FIGO I 0.110; HR PFS FIGO I 0.146 ), and not in advanced stage patients (HR DSS FIGO IV=0.599; HR PFS FIGO IV=0.842).

A subgroup analysis restricted to serous histology was considered but not performed as a significant interaction between serous histology and $\mathrm{p} 53$ could not be demonstrated $(p=0.10)$.

p53 and tumour progression of FIGO stage I patients. A significant association was found between disease progression after primary surgery and accumulation of $\mathrm{p} 53$ protein in the resected tumour tissue $(p=0.0005)($ Table $\mathrm{V})$. As many as $89 \%$ (81/91) of the FIGO stage I patients with low levels of p53 protein failed to show disease progression during followup, in contrast to $56 \%(10 / 18)$ of patients with high levels of p53 protein (Table V). The 18 tumour types that progressed were 3 clear cell carcinomas (p53 $\leq 5 \%$ ), 7 endometrioid adenocarcinomas ( $\mathrm{p} 53 \leq 5 \%=4, \mathrm{p} 53>5 \%=3$ ), 4 well $/$ moderate differentiated serous adenocarcinomas (p53 $\leq 5 \%=2, \mathrm{p} 53$ $>5 \%=2$ ), 3 poorly differentiated serous adenocarcinomas (p53 $\leq 5 \%=1, \mathrm{p} 53>5 \%=2)$ and 1 mucinous adenocarcinoma (p53 $>5 \%)$.

Also, the Kaplan-Meier estimate of PFS, revealed a significantly longer survival of stage I OC patients with low levels of p53 compared to patients with high levels of p53 protein $(p<0.0001)$ (Figure 2).

\section{Discussion}

In line with previous studies, advanced FIGO stage and residual tumour after surgery were significantly associated with poor prognosis, both in terms of OS, DSS and PFS, and remained independent prognosticators in multivariate analysis (3-5, 27-35). Advanced age at diagnosis contributed independently to DSS but not to OS, in agreement or in contrast with the findings of other studies $(5,12,18,27-32$, $34,35)$. Several factors may impact the relationship between age and mortality, like advanced stage at diagnosis, poor physical health, chemotherapy side effects, and a more conservative treatment of older patients (28). Also, poor histological differentiation and elevated serum CA-125 levels 
were accompanied by poor outcome. However, the relationship between CA-125 and outcome was not independent of other pathological factors, as shown in the multivariate analysis. Differentiation grade was not included in the multivariate analysis as serous adenocarcinoma was separated into high- and low-grade carcinomas. Chang et al. recently reported a significant association between high tumour grade and reduced PFS in a cohort of 203 stage IIIIV OC patients (29). Similar results were reported by Shahin et al. in 171 FIGO stage I-IV patients (35). An independent prognostic value of tumour grade has been suggested in some studies $(3,30,31,34)$, but not in other $(29,33)$. This discrepancy is likely to be caused by differences in the number of patients, clinicopathological features and the type(s) of statistical models used.

CA-125 is a glycoprotein released from OC cells, and elevated protein levels are usually found in blood samples from OC patients. Measurement of CA-125 levels in blood samples and its tissue expression in 382 patients from the MALOVA OC study, has shown a significant correlation between serum CA-125 levels and tissue expression of CA125 (36).

Relapse and the development of resistance to therapy are associated with accumulation of mutations in the unstable genome of the tumour cells and represent significant obstacles in the treatment of OC. Attention has therefore been focused on the therapy directed against tumour angiogenesis, as the endothelial cells have a more stable genome than tumour cells (37). Several endothelial-specific markers, including CD31, may be useful in the estimation of tumour vascularity and the monitoring of the effect of antivascular therapeutic drugs (38). A high number of CD31 positive vessels is associated with poor prognosis in $\mathrm{OC}$, suggesting that angiogenesis has a direct impact on growth and metastatic spread $(11,14,16)$. In this study, a significantly higher number of CD31 vessels was correlated with poor differentiation grade but not FIGO stage, residual tumour, serum CA-125 or survival. Stone et al. have reported a high density of CD31 positive vessels in high grade (grade 1-3) OCs, with advanced FIGO stage and residual tumour presence (16). With respect to survival, Lin et al. found that in 77 OC patients, an increased CD31 vessel expression was associated with advanced FIGO stage, residual tumour and poor survival, and a non-significant trend towards poor histological differentiation (14). In a study by Goodheart et al. high microvessel density was found to be associated with advanced FIGO stage and survival in 77 OC patients (17) but experiments by Rubatt and colleagues studying 106 stage III-IV OC patients, did not support the prognostic value of CD31 positive vessel density with respect to stage, grade and residual tumour (15). Some of the discrepancies between studies may be caused by differences in patient numbers and characteristics, as well as methodological differences in
CD31-staining and estimation of vessel density. Another endothelial cell marker (CD105) has been suggested as a good and possibly more specific alternative for the detection of tumour angiogenesis by staining tumour-associated endothelial cells only (18).

The tumour suppressor gene TP53, encoding the cell-cycle regulatory protein $\mathrm{p} 53$, is often mutated in cancers, and therefore p53 alterations as well as the possible clinical impact of $\mathrm{p} 53$ have been extensively reported. Contradictory publications describing the prognostic value of p53 exist and its precise clinical impact is unaddressed $(6,39)$. Mutation frequency of TP53 was $27 \%$ in 124 stage I-IV OC patients from the Danish MALOVA study (6), where stage III-IV patients with a TP53 missense mutation had significantly poorer survival than wild type patients, both in univariate and multivariate analyses (6).

In this study, p53 expression was significantly increased in poorly differentiated serous adenocarcinoma and in tumours from patients with advanced FIGO stage, with nonradical surgery and high serum CA-125. Furthermore, p53 overexpression predicted reduced DSS and PFS in both univariate and multivariate analyses.

High-grade serous tumours are known to have high TP53 mutation frequency and the tumours often overexpress p53 or have no staining for p53 (40). The high-grade serous tumours are highly aggressive and have a poor prognosis (41). Poor prognosis of high-grade serous tumours was confirmed in our study where a low hazard ratio was found for serous adenocarcinoma well/moderately differentiated, clear cell, endometrioid, mucinous and non-epithelial tumours compared to poorly differentiated serous adenocarcinoma tumours.

Some of the discrepancies between reports describing the prognostic impact of p53 may be due to the use of different strategies for the estimation of p53 alterations. Refinement of the scoring method for p53 detection has been suggested by Yemelyanova et al. (42). Additional literature information regarding p53 in OC patients is available on request (corresponding author).

Interestingly, a significant interaction between p53 expression and FIGO stage was found, disclosing that p53 protein levels have an increased negative impact on DSS and PFS in FIGO stage I compared to FIGO stages II, III and IV. Examination of FIGO stage I patients not receiving adjuvant chemotherapy after primary surgery showed that patients experiencing tumour progression after primary operation had varying p53 status. On the other hand, as many as $89 \%$ of the patients with no progression after primary operation had low expression of the $\mathrm{p} 53$ protein, suggesting that p53 status may have clinical value for low risk patients. Only 10 patients (11\%) of the low-risk patient group, who had low p53 expression, experienced tumour progression, whereas 81 patients $(89 \%)$ had no tumour progression. Therefore, it may be speculated that p53 could be helpful for stratifying FIGO 
stage I patients into a p53 high-risk group that may need additional treatment after primary operation to avoid relapse, and a low-risk patient group (p53 $\leq 5 \%$ ) which is most often cured without the use of adjuvant chemotherapy. A careful follow-up of this low-risk patient group may subsequently identify the minority of patients that progress despite a favourable p53 status and initiate therapeutic treatment.

\section{Acknowledgements}

The Authors thank all colleagues of gynaecology and pathology departments for skilful assistance and for their valuable contribution for this paper. The Authors are grateful to Mel C. Heeran for providing technical assistance and academic talks regarding antibodies and immunohistochemical analysis. This work was supported by grants from Mermaid 1, The Danish Cancer Society and National Cancer Institute, Bethesda, U.S.A. (RO1 CA 61107).

\section{References}

1 Siegel R, Naishadham D and Jemal A: Cancer statistics, 2012. CA Cancer J Clin 62(1): 10-29, 2012.

2 Seidman JD and Kurman RJ: Pathology of ovarian carcinoma. Hematol Oncol Clin North Am 17(4): 909-925, vii, 2003.

3 Steffensen KD, Waldstrom M, Grove A, Lund B, Pallisgard N and Jakobsen A: Improved classification of epithelial ovarian cancer: results of 3 danish cohorts. Int J Gynecol Cancer 21(9): 1592-1600, 2011

4 Wimberger P, Wehling M, Lehmann N, Kimmig R, Schmalfeldt B, Burges A, Harter P, Pfisterer J and du Bois A: Influence of residual tumor on outcome in ovarian cancer patients with FIGO stage IV disease: an exploratory analysis of the AGO-OVAR (Arbeitsgemeinschaft Gynaekologische Onkologie Ovarian Cancer Study Group). Ann Surg Oncol 17(6): 1642-1648, 2010.

5 Hannibal CG, Cortes R, Engholm G and Kjaer SK: Survival of ovarian cancer patients in Denmark: excess mortality risk analysis of five-year relative survival in the period 1978-2002. Acta Obstet Gynecol Scand 87(12): 1353-1360, 2008.

6 Hogdall EV, Kjaer SK, Blaakaer J, Christensen L, Glud E, Vuust $\mathrm{J}$ and Høgdall CK: P53 mutations in tissue from Danish ovarian cancer patients: from the Danish "MALOVA" ovarian cancer study. Gynecol Oncol 100(1): 76-82, 2006.

7 Raspollini MR and Taddei GL: Tumor markers in ovarian carcinoma. Int J Gynaecol Obstet 97(3): 175-181, 2007.

8 Hanahan D and Weinberg RA: The hallmarks of cancer. Cell 100(1): 57-70, 2000

9 Merritt WM and Sood AK: Markers of angiogenesis in ovarian cancer. Dis Markers 23(5-6): 419-431, 2007.

10 Darai E, Bringuier AF, Walker-Combrouze F, Fauconnier A, Couvelard A, Feldmann G, Madelenat P and Scoazec JY: CD31 expression in benign, borderline, and malignant epithelial ovarian tumors: an immunohistochemical and serological analysis. Gynecol Oncol 71(1): 122-127, 1998.

11 Alvarez AA, Krigman HR, Whitaker RS, Dodge RK and Rodriguez GC: The prognostic significance of angiogenesis in epithelial ovarian carcinoma. Clin Cancer Res 5(3): 587-591, 1999.

12 Goodheart MJ, Ritchie JM, Rose SL, Fruehauf JP, De Young BR and Buller RE: The relationship of molecular markers of p53 function and angiogenesis to prognosis of stage I epithelial ovarian cancer. Clin Cancer Res 11(10): 3733-3742, 2005.

13 O'Toole SA, Sheppard BL, Laios A, O'Leary JJ, McGuinness $\mathrm{EP}, \mathrm{D}^{\prime}$ Arcy $\mathrm{T}$ and Bonnar $\mathrm{J}$ : Potential predictors of chemotherapy response in ovarian cancer--how do we define chemosensitivity? Gynecol Oncol 104(2): 345-351, 2007.

14 Lin YG, Han LY, Kamat AA, Merritt WM, Landen CN, Deavers MT, Fletcher MS, Urbauer DL, Kinch MS and Sood AK: EphA2 overexpression is associated with angiogenesis in ovarian cancer. Cancer 109(2): 332-340, 2007.

15 Rubatt JM, Darcy KM, Hutson A, Bean SM, Havrilesky LJ, Grace LA, Berchuck A and Secord AA: Independent prognostic relevance of microvessel density in advanced epithelial ovarian cancer and associations between CD31, CD105, p53 status, and angiogenic marker expression: A Gynecologic Oncology Group study. Gynecol Oncol 112(3): 469-474, 2009.

16 Stone PJ, Goodheart MJ, Rose SL, Smith BJ, DeYoung BR and Buller RE: The influence of microvessel density on ovarian carcinogenesis. Gynecol Oncol 90(3): 566-571, 2003.

17 Goodheart MJ, Vasef MA, Sood AK, Davis CS and Buller RE: Ovarian cancer p53 mutation is associated with tumor microvessel density. Gynecol Oncol 86(1): 85-90, 2002.

18 Taskiran C, Erdem O, Onan A, Arisoy O, Acar A, Vural C, Erdem M, Ataoglu $\mathrm{O}$ and Guner $\mathrm{H}$ : The prognostic value of endoglin (CD105) expression in ovarian carcinoma. Int $\mathrm{J}$ Gynecol Cancer 16(5): 1789-1793, 2006.

19 McBride OW, Merry D and Givol D: The gene for human p53 cellular tumor antigen is located on chromosome 17 short arm (17p13). Proc Natl Acad Sci USA 83(1): 130-134, 1986.

20 Soussi T: The p53 tumor suppressor gene: from molecular biology to clinical investigation. Ann NY Acad Sci 910: 121-137, 2000.

21 Hogdall EV, Christensen L, Hogdall CK, Blaakaer J, Gayther S, Jacobs IJ, Christensen IJ and Kjaer SK: Prognostic value of estrogen receptor and progesterone receptor tumor expression in Danish ovarian cancer patients: from the 'MALOVA' ovarian cancer study. Oncol Rep 18(5): 1051-1059, 2007.

22 Hogdall EV, Christensen L, Kjaer SK, Blaakaer J, Bock JE, Glud E, Nørgaard-Pedersen B and Høgdall CK: Distribution of HER2 overexpression in ovarian carcinoma tissue and its prognostic value in patients with ovarian carcinoma: from the Danish MALOVA Ovarian Cancer Study. Cancer 98(1): 66-73, 2003.

23 Hogdall EV, Christensen L, Hogdall CK, Frederiksen K, Gayther S, Blaakaer J, Jacobs IJ and Kjaer SK: Distribution of p53 expression in tissue from 774 Danish ovarian tumour patients and its prognostic significance in ovarian carcinomas. APMIS 116(5): 400-409, 2008.

24 Hogdall EV, Ryan A, Kjaer SK, Blaakaer J, Christensen L, Bock JE, Glud E, Jacobs IJ and Høgdall CK: Loss of heterozygosity on the $\mathrm{X}$ chromosome is an independent prognostic factor in ovarian carcinoma: from the Danish "MALOVA" Ovarian Carcinoma Study. Cancer 100(11): 2387-2395, 2004.

25 Heeran MC, Hogdall CK, Kjaer SK, Christensen L, Blaakaer J, Christensen IJ and Hogdall EV: Limited prognostic value of tissue protein expression levels of cyclin E in Danish ovarian cancer patients: from the Danish 'MALOVA' ovarian cancer study. APMIS 120(10): 846-854, 2012.

26 Vojtesek B, Bartek J, Midgley CA and Lane DP: An immunochemical analysis of the human nuclear phosphoprotein p53. New monoclonal antibodies and epitope mapping using recombinant p53. J Immunol Methods 151(1-2): 237-244, 1992. 
27 Skirnisdottir I and Seidal T: Association of p21, p21 p27 and p21 p53 status to histological subtypes and prognosis in low-stage epithelial ovarian cancer. Cancer Genomics Proteomics 10(1): 27-34, 2013.

28 Wong KH, Mang OW, Au KH and Law SC: Incidence, mortality, and survival trends of ovarian cancer in Hong Kong, 1997 to 2006: a population-based study. Hong Kong Med J 18(6): 466-474, 2012.

29 Chang SJ, Bristow RE and Ryu HS: Impact of complete cytoreduction leaving no gross residual disease associated with radical cytoreductive surgical procedures on survival in advanced ovarian cancer. Ann Surg Oncol 19(13): 4059-4067, 2012.

30 Mahdi H, Kumar S, Munkarah AR, Abdalamir M, Doherty M and Swensen R: Prognostic impact of marital status on survival of women with epithelial ovarian cancer. Psychooncology 22(1): 83-88, 2013.

31 Lassus H, Leminen A, Lundin J, Lehtovirta P and Butzow R: Distinct subtypes of serous ovarian carcinoma identified by $\mathrm{p} 53$ determination. Gynecol Oncol 91(3): 504-512, 2003.

32 Garcia-Velasco A, Mendiola C, Sanchez-Munoz A, Ballestin C, Colomer R and Cortes-Funes $\mathrm{H}$ : Prognostic value of hormonal receptors, p53, ki67 and HER2/neu expression in epithelial ovarian carcinoma. Clin Transl Oncol 10(6): 367-371, 2008.

33 Tomic S, Ilic FJ, Babic D, Sundov D, Kuret S and Andelinovic $\mathrm{S}$ : c-erbB-2, p53, and $\mathrm{nm} 23$ proteins as prognostic factors in patients with epithelial ovarian carcinoma. Croat Med J 44(4): 429-434, 2003.

34 Reles A, Wen WH, Schmider A, Gee C, Runnebaum IB, Kilian U, Jones LA, El-Naggar A, Minguillon C, Schönborn I, Reich O, Kreienberg R, Lichtenegger W and Press MF: Correlation of p53 mutations with resistance to platinum-based chemotherapy and shortened survival in ovarian cancer. Clin Cancer Res 7(10): 2984-2997, 2001.

35 Shahin MS, Hughes JH, Sood AK and Buller RE: The prognostic significance of p53 tumor suppressor gene alterations in ovarian carcinoma. Cancer 89(9): 2006-2017, 2000.
36 Hogdall EV, Christensen L, Kjaer SK, Blaakaer J, KjaerbyeThygesen A, Gayther S, Jacobs IJ and Høgdall CK: CA125 expression pattern, prognosis and correlation with serum CA125 in ovarian tumor patients. From The Danish "MALOVA" Ovarian Cancer Study. Gynecol Oncol 104(3): 508-515, 2007.

37 Paley PJ: Angiogenesis in ovarian cancer: molecular pathology and therapeutic strategies. Curr Oncol Rep 4(2): 165-174, 2002.

38 Thaker PH, Yazici S, Nilsson MB, Yokoi K, Tsan RZ, He J, Kim SJ, Fidler IJ and Sood AK: Antivascular therapy for orthotopic human ovarian carcinoma through blockade of the vascular endothelial growth factor and epidermal growth factor receptors. Clin Cancer Res 11(13): 4923-4933, 2005.

39 de Graeff P, Crijns AP, de Jong S, Boezen M, Post WJ, de Vries EG, van der Zee AG and de Bock GH: Modest effect of p53, EGFR and HER-2/neu on prognosis in epithelial ovarian cancer: a meta-analysis. Br J Cancer 101(1): 149-159, 2009.

40 Kohn EC and Ivy SP: Whence High-Grade Serous Ovarian Cancer. Am Soc Clin Oncol Educ Book 37: 443-448, 2017.

41 Battista MJ, Cotarelo C, Almstedt K, Heimes AS, Makris GM, Weyer $\mathrm{V}$ and Schmidt M: Validation of a two-tier grading system in an unselected, consecutive cohort of serous ovarian cancer patients. Arch Gynecol Obstet 294(3): 599-606, 2016.

42 Yemelyanova A, Vang R, Kshirsagar M, Lu D, Marks MA, Shih I and Kurman RJ: Immunohistochemical staining patterns of p53 can serve as a surrogate marker for TP53 mutations in ovarian carcinoma: an immunohistochemical and nucleotide sequencing analysis. Mod Pathol 24(9): 1248-1253, 2011.
Received December 5, 2018

Revised December 14, 2018 Accepted December 17, 2018 